\section{Eleutherodactylus glanduliferoides (La Selle Grass Frog). Conservation.}

Date of observation: 20 November 2009. Location: Haiti: L'Ouest; 5 km S Furcy. Coordinates:18.3949, -72.2409. Elevation: $1586 \mathrm{~m}$. This critically endangered species (IUCN, CR) was last found at this locality on 9 July 1985 but we did not see or hear it calling this evening. Frog activity was reduced, but we encountered E. audanti, E. furcyensis, and E. inoptatus. The rainy season had ended early and conditions were unusually dry, which may explain reduced frog activity. Accompanied by Elisabeth Rochel, Richard Thomas, and Eladio Fernandez. Thanks to Philippe Bayard and the Audubon Society of Haiti.

S. Blair Hedges, Pennsylvania State University, Department of Biology, 208 Mueller Lab, University Park, Pennsylvania, 16802, USA, sbh1@psu.edu.

Citation: Hedges SB. 2010. Eleutherodactylus glanduliferoides (La Selle Grass Frog). Conservation. Caribbean Herpetology 2:1.

Published online 21 August 2010 